\title{
Digital instrument with configurable hardware and firmware for multi-channel time measures
}

Cite as: Rev. Sci. Instrum. 90, 055113 (2019); https://doi.org/10.1063/1.5028131

Submitted: 07 March 2018 . Accepted: 04 May 2019 . Published Online: 29 May 2019

Nicola Lusardi (D), Fabio Garzetti (D), and Angelo Geraci (D)
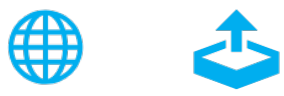

\section{ARTICLES YOU MAY BE INTERESTED IN}

A 6.6 ps RMS resolution time-to-digital converter using interleaved sampling method in a $28 \mathrm{~nm}$ FPGA

Review of Scientific Instruments 90, 044706 (2019); https://doi.org/10.1063/1.5084014

A scalable arbitrary waveform generator for atomic physics experiments based on fieldprogrammable gate array technology

Review of Scientific Instruments 90, 043101 (2019); https://doi.org/10.1063/1.5051124

A multifunction digital receiver suitable for real-time frequency detection and compensation in fast magnetic resonance imaging

Review of Scientific Instruments 90, 053707 (2019); https://doi.org/10.1063/1.5092312

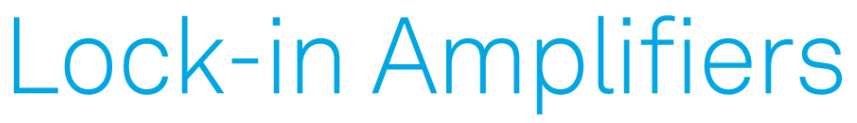

... and more, from DC to $600 \mathrm{MHz}$ watch 


\title{
Digital instrument with configurable hardware and firmware for multi-channel time measures
}

\author{
Cite as: Rev. Sci. Instrum. 90, 055113 (2019); doi: 10.1063/1.5028131 \\ Submitted: 7 March 2018 - Accepted: 4 May 2019 • \\ Published Online: 29 May 2019
}

Nicola Lusardi, ${ }^{\text {a) }}$ (D) Fabio Garzetti, ${ }^{\text {b) }}$ (D) and Angelo Geraci ${ }^{c)}$ (D)

\begin{abstract}
AFFILIATIONS MI 20133, Italy

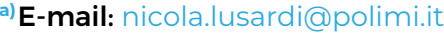

b) E-mail: fabio.garzetti@polimi.it

${ }^{c}$ E-mail: angelo.geraci@polimi.it
\end{abstract}

Politecnico di Milano, Department of Electronics, Information and Bioengineering_DEIB, Via Camillo Golgi 40, Milan,

\begin{abstract}
A new digital instrument for timing of events is presented. It is based on a reconfigurable, high-performance, 16-channel time-to-digital converter implemented in a Xilinx 7 Series $28-\mathrm{nm}$ field programmable gate array device. Each channel provides timestamps with a least significant bit of 2 ps that states the resolution, whereas instead the single-shot precision is below 12.5 ps rms with the possibility of multihit measures at the maximum rate of $20 \mathrm{MHz}$. The default width of the full-scale range is $157 \mu$ s that can be extended at users choosing up to 15 days by means of proper time tagging procedures made available. The instrument achieves performance in terms of precision, resolution, and full-scale range of measurement at the state-of-art of existing solutions. The novelty is that besides performance, the presented instrument is totally reconfigurable by the user both in the hardware and in the firmware parts. Moreover, novel techniques of event acquisition (e.g., level-zero trigger) are introduced. These further features are not present in any other instrument available nor in the literature or in commerce and constitute a difference with respect to all referenced instruments.
\end{abstract}

Published under license by AIP Publishing. https://doi.org/10.1063/1.5028131

\section{INTRODUCTION}

The time-to-digital converter (TDC) processing core is based on Tapped Delay-Line (TDL) and instantiated as IP-core module compatible with Xilinx's Field Programmable Gate Arrays (FPGAs) and Systems on Chips (SoCs) of last generation.

The instrument structure is composed of hardware, firmware, and software parts. Three acquisition ways are available for managing properly the timestamps, which fit at best with the philosophy of the applications the TDCs are devoted to. The Level-Zero Trigger (LZT) is a novel technique that allows collecting only the events that follow a user defined pattern. For guaranteeing a continuous acquisition of absolute timestamps with a large range (up to 15 days), a Time-Tagging Mode (TTM) is available. Furthermore, a lite version of the TTM called MultiHits Acquisition (MHA) is integrated; in this sense, it is possible to acquire absolute time measures of several events into a dynamic range of $157 \mu$ s with respect to a trigger signal provided by the user.

The instrument can be reconfigured by the final user both in firmware and hardware parts. In particular, the firmware and the software associated with the hardware are modularly structured so that the processing is optimized by partitioning the computing tasks in parallel segments.

All characteristics of the instruments have been experimentally validated. In Sec. IV, we do not describe experiments in detail, in order to limit the length of the manuscript. In fact, we favor the discussion of the results of the experiments with the instruments under comparison.

The extremely wide evidence of applications, where the timing of signals is a fundamental request both as single and especially multiple simultaneous measures, is continuously promoting the development of innovative techniques and the design of devices for their implementation.

The process of measurement consists in recognizing events and providing their timestamp, i.e., the representation of the time they occur. Since this timestamp is always referred to a reference instant, in practice, the measurement consists in calculating the time interval between a couple of "start" and "stop" instants. Furthermore, the timing measures are not used only for direct evaluations, indeed there are many physical phenomena observed in an indirect way. 
So, a time interval can represent a space distance in Time-of-Flight (ToF) applications ${ }^{2}$ or can be used for extracting the spectrum of a material in Time-Correlated Single Photon Counting (TCSPC) $)^{3,4}$ and in Time-of-Flight Mass-Spectroscopy (ToF-MS) ${ }^{5}$ or for getting the fluorescence of a substance by means of Fluorescence Spectroscopy (FS). ${ }^{6}$ Moreover, the concept of ToF can be extended in bi- and tri-dimensional imaging. Time-of-Fly Laser-Raging (ToFLR) ${ }^{7}$ and Time-of-Fly Positron Emission Tomography (ToF-PET) are the most significant examples.

For completeness, we have to say that a new generation of electronic devices, such as Analog-to-Digital Converters (ADCs) ${ }^{9}$ and Phase-Locked Loops (PLLs), ${ }^{10}$ have been redesigned using the "time-mode" approach. Here, developing new time-interval-meters with higher and higher performance allows us to increase the quality of the measures so improving the result of the application in which they are used. Historically, the well-established method for highaccuracy measurement of time intervals is the Time-to-Amplitude Conversion (TAC). ${ }^{11,12}$ However, nowadays in the digital era, the timing information can be converted directly in a digit without using any analog circuit. These systems, called Time-to-Digital Converters (TDCs), are the landmark for determining the time instants at which digital events take place. Nowadays, TDCs are the building blocks of "time-mode" circuits. ${ }^{13}$ These circuits code of information by measuring the time instants at which the electrical signals (voltage and current) cross reference values, which is exactly the opposite of what happens in voltage and current mode circuits. Therefore, time measurement is at the basis of this new philosophy of processing. The key point is that time-mode circuits benefit at most from technology scaling. ${ }^{14-16}$ In fact, the switching characteristics of MOS transistors are improved to such an extent that the time resolution of digital circuits decisively surpasses the resolution of analog ones implemented in active devices scaled in the nanometer range. Moreover, it is important to mention that the analog circuits implemented by such small devices usually have quite poor performance. Moreover, since fully digital, TDCs offer features that are not possessed by either voltage-mode or current-mode options. ${ }^{17}$ Among them, full programmability, tunable characteristics, easiness of portability, low-power consumption, high-speed operation, lower migration cost from one generation of technology to another, less sensitivity to interferences such as cross talk, switching noise, and substrate coupling are of primary importance, which have a detrimental impact on the performance of either voltage and current-mode circuits.

Since a few years, digital programmable logic devices have made feasible the implementation of synchronous and lowperformance TDC architectures. ${ }^{18}$ Today, the last generation of FPGAs and SoCs are characterized by lower and lower propagation delays that make feasible the realization of high-resolution and high-rate asynchronous TDCs. Moreover, the abundance of logic resources allows the implementation of more and more complex and effective calibration algorithms that allow achieving performance such as precision, accuracy, and temperature stability comparable with Application Specific Integrated Circuit (ASIC) realizations at similar technological nodes. ${ }^{19-21}$ In this way, all the well-known advantages of using programmable devices are exploited, such as reduced time-to-market and easier migration of processing architectures among devices, just to name a few in addition to the general advantages of the digital systems mentioned above. In particular, the implementation of multichannel architectures becomes straightforward while keeping on foreground the functional performance. ${ }^{22-24}$ This leads to a new generation of tunable Hardware Description Language (HDL) TDCs where the user can easily customize the system inside some range of choices, maintaining at the same time the portability between different programmable devices. $^{25,26}$

As important as the architecture and the relative performance is the management of the timestamps produced by the TDC that needs different acquisition modes tailored on specific application targets. Well-known examples in the literature are the "time-tagging" mode that allows us to get measures at a relative low rate for long time ${ }^{27}$ and several multihit solutions that permit to acquire relative few events at a high rate. ${ }^{28}$

Generally, in a physical experiment based on high-performance time measures, after the data collection (e.g., "time-tagging," multihit) all timestamps are elaborated in the postprocessing mode. In this way, a huge amount of memory and bandwidth to store and route the information is mandatory due to the necessity of all timestamps to have a high-rate and large number of bits (high-resolution over a wide dynamic-range) for guaranteeing high-performance. However, only a restricted number of these timestamps are correlated and represent the observed event, while the main part of them is due to random events. For this reason, a novel technique called LevelZero Trigger (LZT) is proposed. With respect to the "time-tagging" and multihit solutions, the LZT allows filtering at the firmware level all the timestamps in real-time, acquiring only those that follow a user defined pattern that models the time response of the physical event. In this way, it is possible to discard useless data with important memory and bandwidth saving. ${ }^{29,30}$

This work deals with the design and realization of a full programmable and multichannel instrument for timing of signals, which is based on a recent TDC architecture tailored for the last generation FPGA devices as IP-core. The instrument structure is a hardware, firmware, and software bundle for high-performance time measures in experiments where very fine time resolving is a primary request. The synergy of firmware and software maximizes the performance of the instrument also in real-time applications in terms of high-resolution and high-precision over a wide full-scale range, but also includes features like high full-scale range, high acquisition rate, thermal stability, real-time processing, flexibility, modularity, portability, low time-to-market, and at last controlled implementation costs. $^{31-33}$

\section{TDC IP-CORE}

Programmable logic devices, especially FPGA and SoC, allow us to implement TDCs with different kinds of structures. Looking into the literature, we can roughly divide these architectures between TDCs based on feedback (FB) circuits, ${ }^{34,35}$ e.g., ring-oscillators, and feedforward (FF) ones based on delay-lines. ${ }^{34}$

The high difference of propagation delay values among combinatorial logic elements, mainly due to the scaling down of technology, favors the implementation of feedforward solutions with respect to the feedback ones. To confirm this, we can consider the worsening in resolution from 5 ps to 63.3 ps shown by two TDCs based on a ring-oscillator implemented in $28-\mathrm{nm}^{34}$ and $90-\mathrm{nm}^{35}$ technology, respectively. Differently, a TDC based on delay-line offers a resolution of $75 \mathrm{ps}$ in $130-\mathrm{nm},{ }^{36} 50 \mathrm{ps}$ in $90-\mathrm{nm},{ }^{37}$ and 
TABLE I. Comparison of the resolution of the proposed TDC IP-core with respect to architectures present in the literature of TDCs implemented in FPGA. FB and FF stand for "feedback" and "feedforward," respectively.

\begin{tabular}{lcccc}
\hline \hline & Resolution (LSB) (ps) & Architecture & Technology (nm) & Subinterpolation \\
\hline Reference 34 & 5 & FB & 90 & No \\
Reference 35 & 63.3 & FB & 28 & No \\
Reference 36 & 75 & FF & 130 & No \\
Reference 39 & 10 & FF & 130 & (Average) \\
Reference 37 & 50 & FF & 90 & No \\
Reference 38 & 30 & FF & 65 & No \\
Proposed TDC & 2 & FF & 28 & Yes \\
\hline \hline
\end{tabular}

30 ps in $65-\mathrm{nm}^{38}$ technology. Moreover, to overcome the limit of resolution due to the minimum propagation delay of the logic, further mechanisms of averaging and calibration are inserted into delay-line TDC architectures, lowering the resolution down to $10 \mathrm{ps}$ in a 130 -nm FPGA device.

The different propagation times of signals through the cascaded bins constituting a delay-line implemented in a FPGA device causes not uniform quantization. ${ }^{40}$ These differences, in the case of averaging, have a negative impact on resolution and precision and severely limit the linearity. The slowest bins, called ultrabins, are main responsible for this. As a consequence, subinterpolation algorithms and dynamic calibration procedures are mandatory, for reducing the effect of ultrabins and improving the linearity. These evidence are summarized in Table I.

Precisely, the subinterpolation consists in merging different measurements coming from several TDLs composed of "real bins." In this way, it is possible to obtain a Virtual Tapped Delay-Line (V-TDL) composed of "virtual bins" characterized by a faster propagation delay. Different from a standard average process on a not uniform quantization, if one of the measures falls in the ultrabin, this does not limit the resolution. ${ }^{41}$ Furthermore, the dynamic calibration allows compensating both the nonlinearity of the delay-line and its thermal drift.

The implemented TDC IP-core is a user friendly and multiplatform module. It has 16 channels and provides high-resolution and high-precision by means of the Super Wave Union (SuperWU) subinterpolation $^{39,42,43}$ of each channel, whereas the Nutt interpolation allows a wide full-scale range and a multichannel structure.

The measurement of the time interval limited by start event $i$ and stop event $j, T_{j i}$, is calculated as the difference between the timestamps $T_{i}$ and $T_{j}$ as shown in Fig. 1,

$$
T_{j i}=T_{j}-T_{i} .
$$

According to the Nutt technique of interpolation, the generic timestamp $T_{i}$ is composed of a "coarse" $\left(T_{i}^{C O A R S E}\right)$ and a "fine" $\left(T_{i}^{F I N E}\right)$ contribution (Fig. 1), i.e.,

$$
T_{i}=T_{i}^{C O A R S E}-T_{i}^{F I N E}
$$

For doing this, the IP-core implements two different types of TDCs (Fig. 2). A synchronous Coarse-Counter (CC) returns the coarse part $T_{i}^{C O A R S E}$ of the timestamp by counting the number of clock periods between $i$ and the reference zero time, i.e., the output of

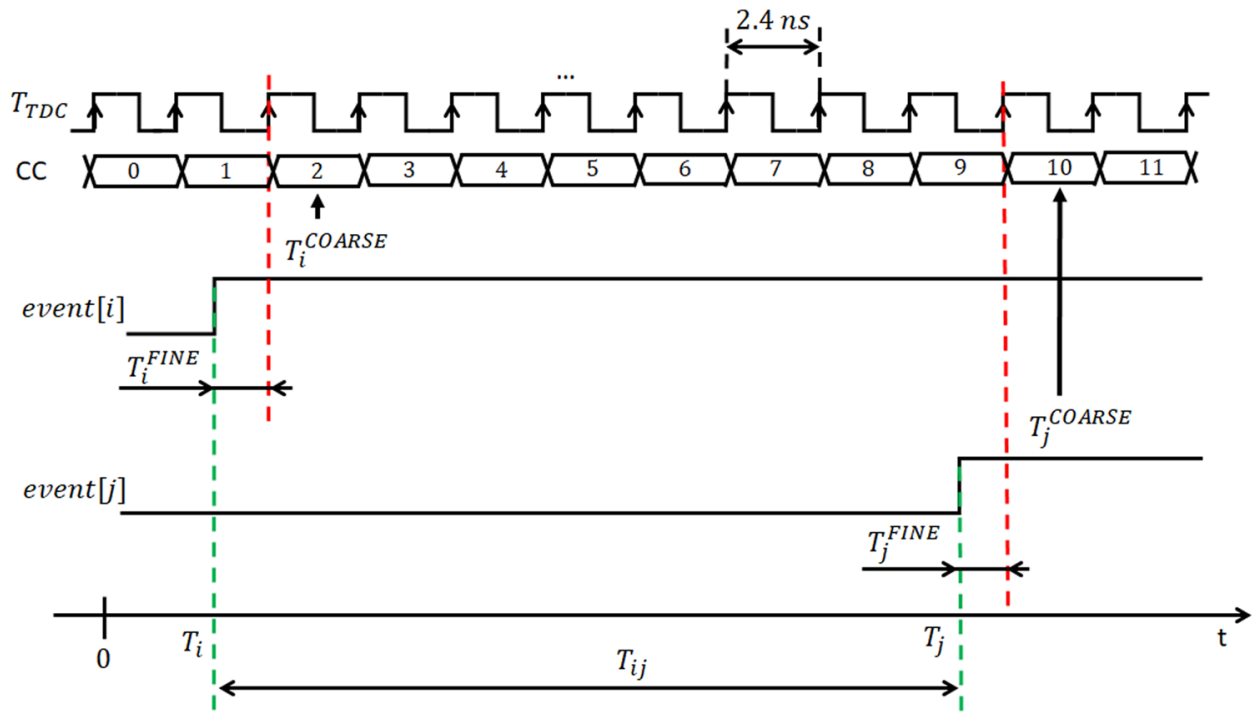

FIG. 1. Timing diagram of the calculation procedure of the timestamps $T_{i}$ and $T_{j}$ of the events $i$ and $j$ using the Nutt technique of interpolation. 


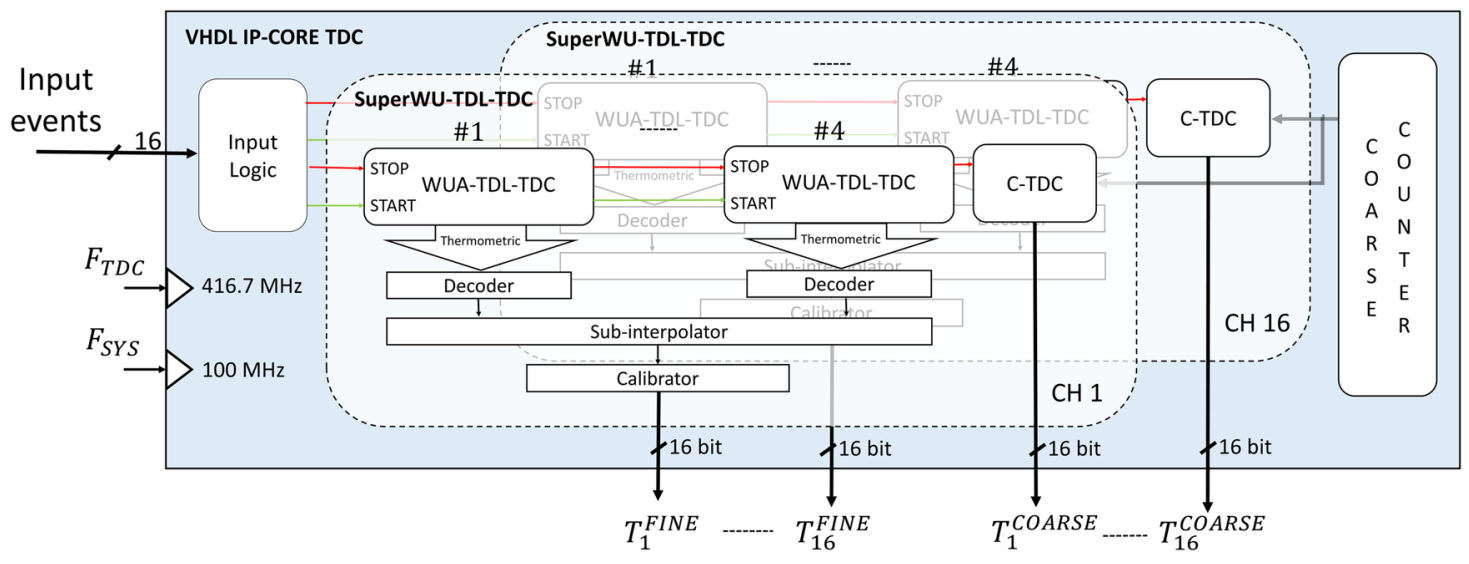

FIG. 2. Block diagram of the TDC IP-core, where the two types of TDCs implemented, C-TDC and TDL-TDC, are put in evidence. In the TDL-TDC block, the implemented subinterpolation method, in this case wave union $\mathrm{A}$, is also specified.

the Counter-TDC (C-TDC). The fine part of the timestamp $T_{i}^{F I N E}$ is measured by a subinterpolated Tapped Delay-Line TDC (TDLTDC) as the difference between the distance of the event $i$ from the following clock edge. ${ }^{32}$ The number of bits of the coarse-counter determines the full-scale range of the instrument.

So, the fine contribution is provided by a Super Wave Union Tapped Delay-Line TDC (SuperWU-TDL-TDC) ${ }^{43}$ synchronized with the C-TDC. The SuperWU-TDL-TDC implements the subinterpolation of 4 Wave Union A Tapped Delay-Line TDCs (WUATDL-TDC) ${ }^{19}$ that performs 2 measurements in parallel for a total of 8 fine measurements for each single event on the relative channel. ${ }^{4}$

The implemented subinterpolation algorithm speeds up the TDL, thanks to the parallel structure that makes it equivalent to a Virtual Tapped Delay-Line (V-TDL) composed of 1200 virtual bins instead of 150 real ones, which are, in average, 8 times faster. As negative by-side effect, this procedure adds a further jitter component called equivalent-time noise, ${ }^{43}$ which increases with the subinterpolation order and is caused by the mechanism of generation of the replicas for the multiple measures performed. In this case, an improvement of a factor 8 is enough to achieve a resolution [least significant bit (LSB)] of 2 ps while keeping negligible the equivalenttime noise. This guarantees single channel precision $\left(\sigma_{T D C}\right)$ below 9.5 ps rms. This characterization has been performed using a pure digital signal, with a negligible jitter, at the input of the TDC-IP.

TABLE II. Comparison of the most relevant figures of merit of the proposed TDC IPcore with respect to TDCs at the state-of-the-art implemented in FPGA proposed in the literature.

\begin{tabular}{lcccc}
\hline \hline & $\begin{array}{c}\text { Resolution } \\
\text { (LSB) (ps) }\end{array}$ & $\begin{array}{c}\text { Precision } \\
\text { (ps rms) }\end{array}$ & $\begin{array}{c}\text { INL } \\
\text { (ps) }\end{array}$ & $\begin{array}{c}\text { IP-core } \\
\text { organization }\end{array}$ \\
\hline Proposed TDC & 2 & 9.7 & 4.2 & Yes \\
Reference 42 & N.A. & 8.7 & N.A. & No \\
Reference 56 & 2.93 & 4.2 & 11.7 & No \\
Reference 57 & 3.29 & 4.2 & 6.58 & No \\
Reference 58 & 2.5 & 6.7 & 7.5 & No \\
\hline \hline
\end{tabular}

The synchronous parts of the TDC logic, such as dynamic "bin-by-bin" calibration, ${ }^{19,20}$ decoding, ${ }^{44}$ and ancillary finite-state machines, run at the system clock $\left(f_{C L K}\right)$ of $100 \mathrm{MHz}$. Each single channel achieves a maximum rate of measure of $20 \mathrm{MHz}$ with power consumption below $13.8 \mathrm{~mW}$, using 3975 flip-flops (FFs), 3684 Look-Up Tables (LUTs), 1 DSP, and 54-kbit BRAM, which is in the first approximation an occupancy below $1 \%$ of the available resources in the considered device.

As Table II shows, the proposed HDL module achieves performance comparable to the state-of-the-art of ASIC and FPGA solutions. Furthermore, the organization of the firmware as an IPcore allows easily the migration of the TDC among all last generation Xilinx FPGAs and SoCs, that is, Series-7, UltraSCALE and UltraSCALE+.

\section{INSTRUMENT STRUCTURE}

The instrument is composed of the analog front-end for discriminating time events, which allows the simultaneous acquisition of up to 16 events carried in by single-ended signals, the processing section programmed with the custom firmware that allows different acquisition modes, various communication modules (e.g., USB 3.0 and Ethernet links), and the software running on a generic computer (Fig. 3).

\section{A. Analog-to-digital conversion of events}

The role of the analog front-end is to convert into digital pulses the time events present in input analog signals. The discriminator of events is an ultrafast and low-jitter comparator (Analog Devices ADCMP607BCPZ ${ }^{45}$ ), whose output is a logic level in the Low-Voltage Differential Signal (LVDS) format. The user can set the threshold of the comparator with a resolution of $50 \mu \mathrm{V}$ by means of two 16-bit and 8-channel I ${ }^{2} \mathrm{C}$-programmable DACs (Analog Devices AD5675R ${ }^{46}$ ). Moreover, the user can switch from DC to AC coupling through a tunable high-pass filter if the rejection of a bias is requested.

The analog section is designed for minimizing any effect of cross talk by means of proper routing rules based on fully 


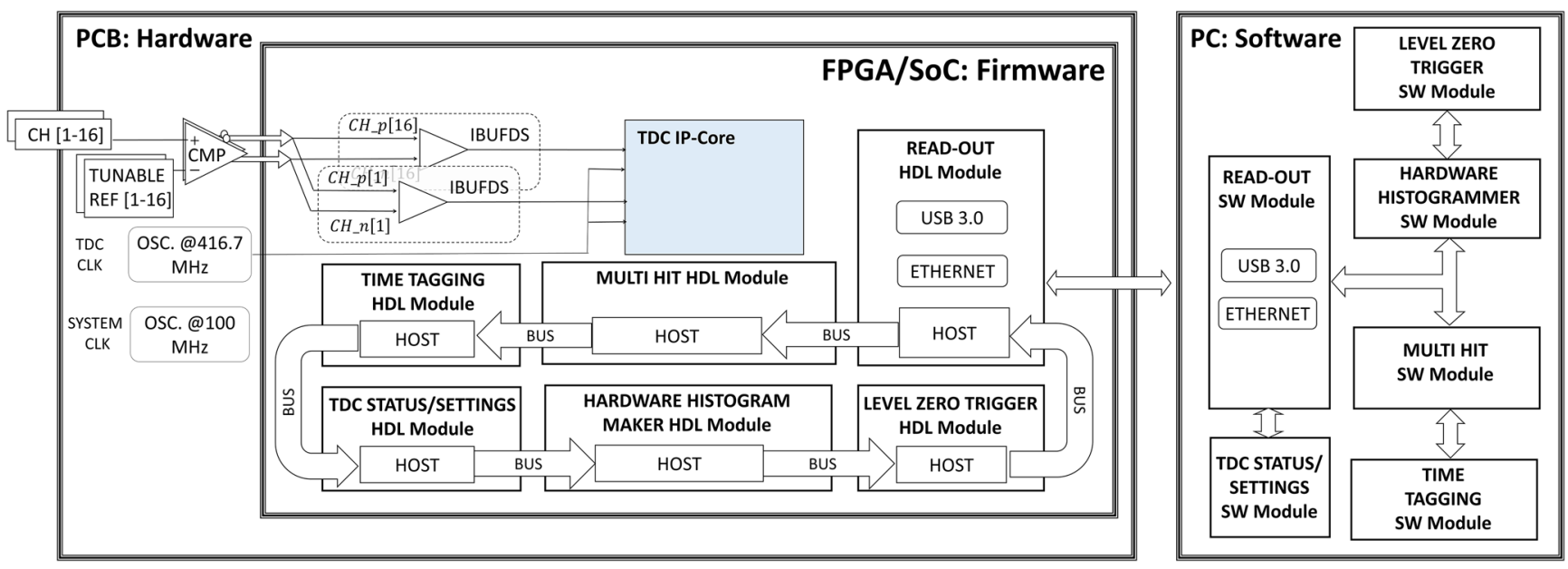

FIG. 3. Schematic overview of the complete structure of the instrument. The shaded region highlights the position of the TDC IP-core.

differential connections, isolation among traces, and impedance matching. Decoupling between analog and digital sections guarantees very low ground bounce that minimizes the threshold jitter during the operation of comparison. This allows us to achieve a contribution of timing jitter $\left(\sigma_{J}\right)$ lower than 7 ps rms, which has been calculated by subtracting the precision obtained with a pure digital signal $\left(\sigma_{T D C}\right)$ to the precision of the measurement achieved by a weak analog signal with $10 \mathrm{~ns}$ rising edge and $200 \mathrm{mV}$ maximum amplitude,

$$
\sigma_{J}=\sqrt{\sigma_{C H}^{2}-\sigma_{T D C}^{2}}
$$

\section{B. Processing unit and firmware}

In order to offer the maximum versatility of the system, the processing section of the instrument has been designed as a carrier of replaceable micromodules equipped with different Xilinx FPGAs and SoCs. ${ }^{48}$ In this way, the migration of the custom modular firmware is feasible through all Xilinx 7 Series, UltraScale and UltraScale+ FPGAs and SoCs.

Therefore, the proposed instrument is reconfigurable in a sense of both of firmware and hardware.

The instrument configuration here considered (Fig. 4) is equipped with the module TE0712-02-200-2C by Trenz Electronics $^{47}$ that hosts an industrial 28-nm programmable Xilinx Artix ${ }^{\circledR}-7$ XC7A200T-1 FPGA (33 650 SLICEs, 13 140-kbit BRAM, 740 DSPs) in package of area $19 \times 19 \mathrm{~mm}^{2}$.

In the following, we focus on the most relevant features of the firmware highlighting the factors that primarily influence the performance of the instrument and are under user's control.

The firmware is organized as a daisy chain bus with a high grade of modularity, where the TDC IP-core calculates the timestamps and other ancillary modules perform their real-time processing (Fig. 3). The read-out module, which is the default master of the bus, implements USB 3.0 and Ethernet interfaces. Timestamps from the TDC IP-core are sent to one or more acquisition modules, which have custom packagers that send data to the host PC. Packagers and read-out stage communicate using the daisy chain bus at the rate of $80 \mathrm{MB} / \mathrm{s}$. Also, the user's settings are sent from the host PC to the processing unit through the daisy chain bus.

One of the most significant degrees of freedom at user's availability is the set of ways of acquisition of the timestamps, i.e., LevelZero Trigger (LZT), Time-Tagging Mode (TTM), and MultiHits Acquisition (MHA). Each one of these ways has been developed for maximizing the "acquisition efficiency." In this sense, the LZT allows filtering in FPGA and acquiring in software only the timestamps that follow a specified pattern; the TTM acquires all the timestamps extending the dynamic range up to some days without the impact on the resolution; the MHA permits to work at the maximum possible rate acquiring timestamps with the best resolution inside the dynamic range.

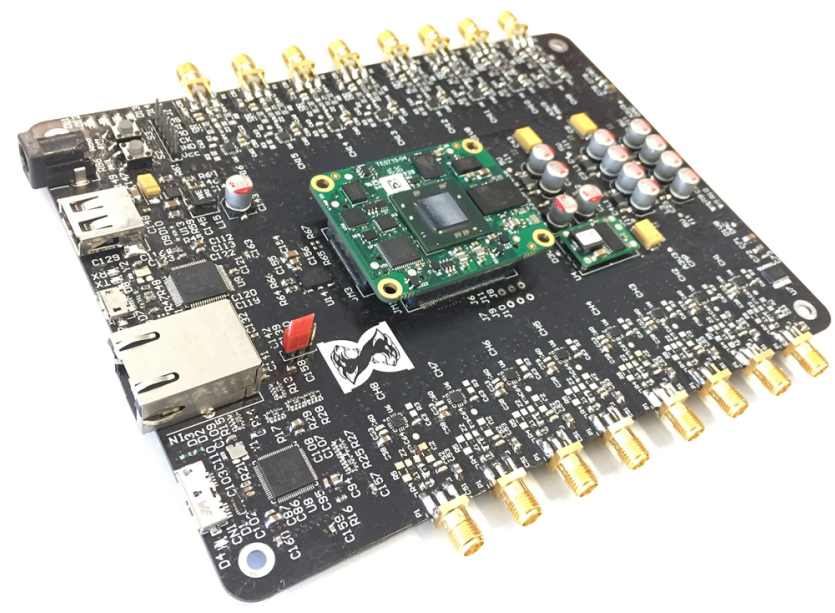

FIG. 4. Picture of the instrument with the piggyback processing micromodule mounted in the middle through connectors. Changing this module allows also the hardware reconfiguration of the instrument. 
The level-zero trigger module is devoted to collect only the measures of events whose sequences of start and stop instants follow a programmable pattern in a defined time window. In run-time and via software, the user can set all start and stop conditions and the acquisition windows with a resolution of $10 \mathrm{~ns}$. Therefore, it is possible to discard inside the FPGA the events that are not compliant with the specified pattern. For instance, this technique is suited for discriminating real events from dark counts, thus improving the efficiency. ${ }^{30}$

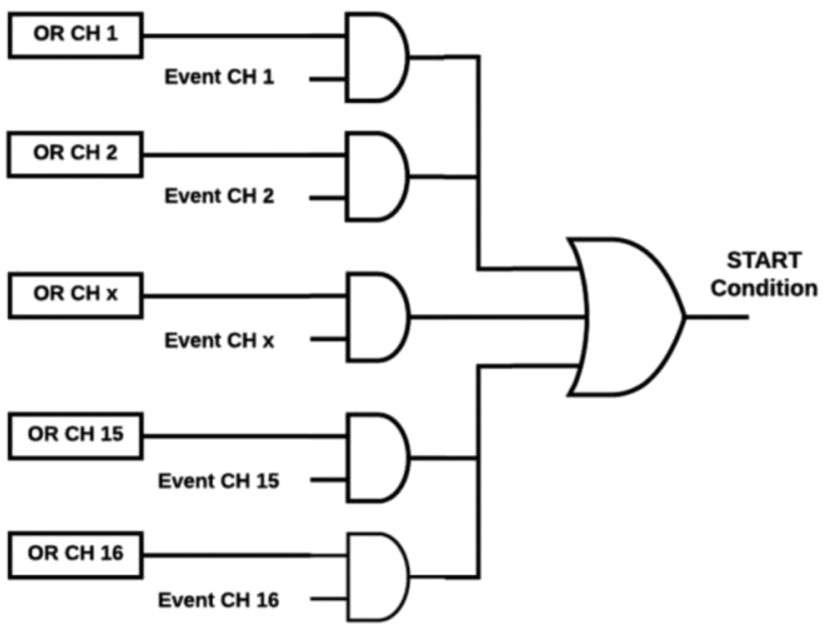

In this way, the bandwidth of the interconnection between TDC and PC and the storage memory are only occupied by those timestamps that are filtered by the firmware.

The user can set in run-time all the start and stop conditions, by means of the so-called AND-mask and OR-mask (Fig. 5), and the maximum measure interval between them. The timestamps filtered by the level-zero trigger are sent to the $\mathrm{PC}$ at a maximum

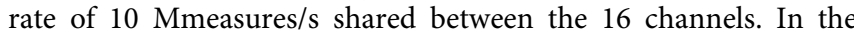
LZT, the concept of "acquisition efficiency" consists in minimizing

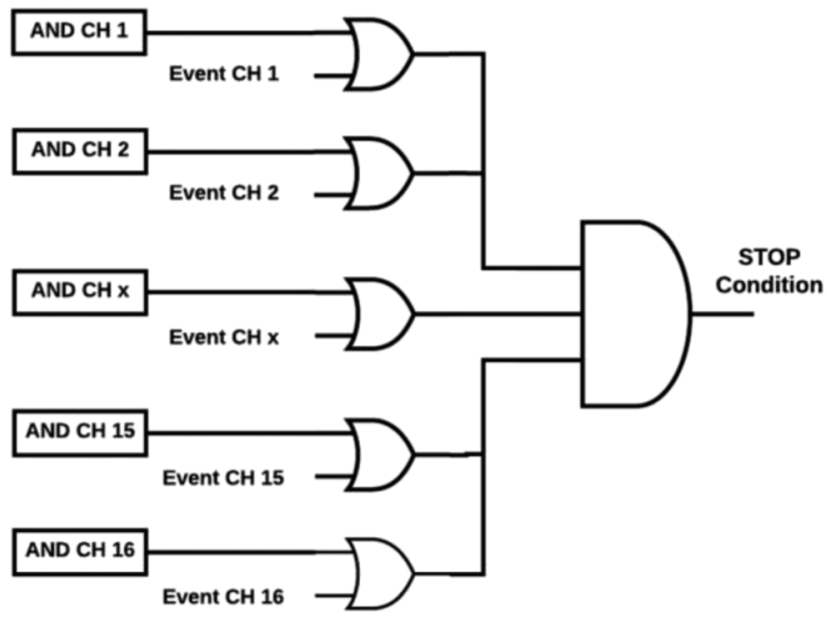

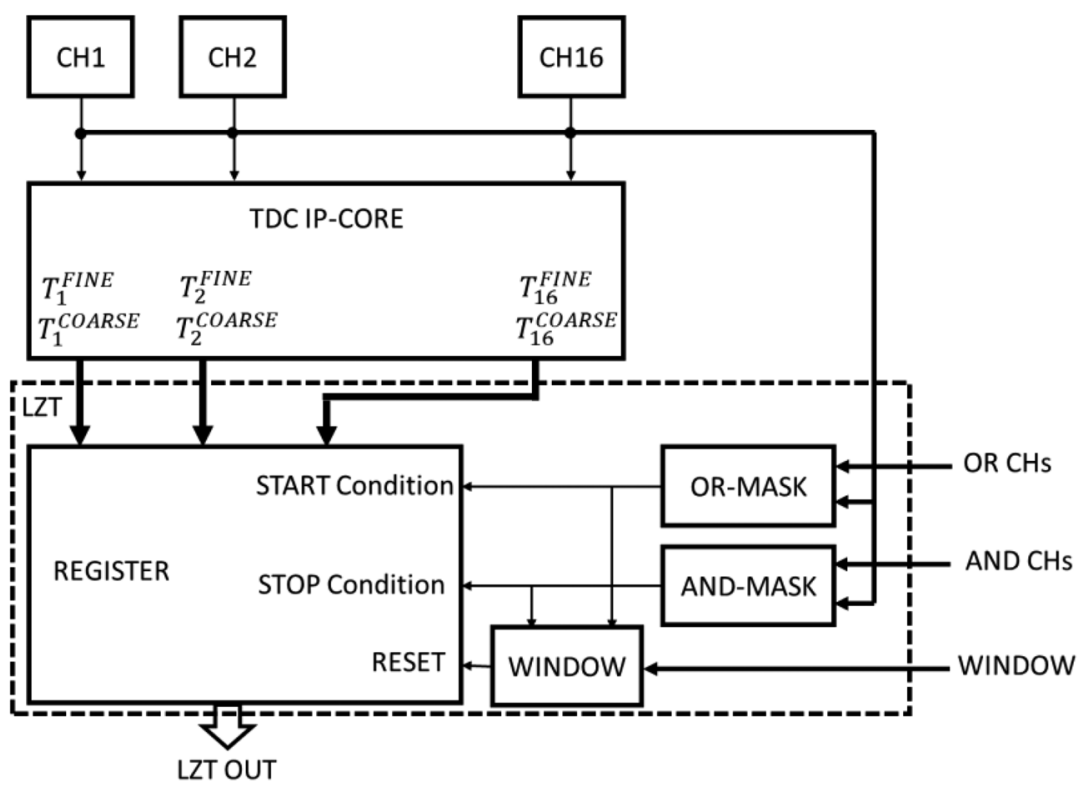

FIG. 5. If an event at the generic $x$ input can start a measurement, its corresponding position in the OR-mask (OR CHx) is set equal to "1" (top left). This is sent to a 2-input-AND gate together with the $x$ input signal. When the AND condition is true, the output of the OR gate, collecting all AND-gate outputs, goes high and the LZT starts to store timestamps (bottom). If the generic $x$ input has to be present for the validity of the measurement, its corresponding position in the AND-mask (AND $\mathrm{CH} x$ ) is set equal to " 0 " (top right). This is sent to a 2-input-OR gate together with the real $x$ input signal. The collected timestamps are considered valid if all events having "0" in the AND-mask arrived before the end of the window (bottom). 


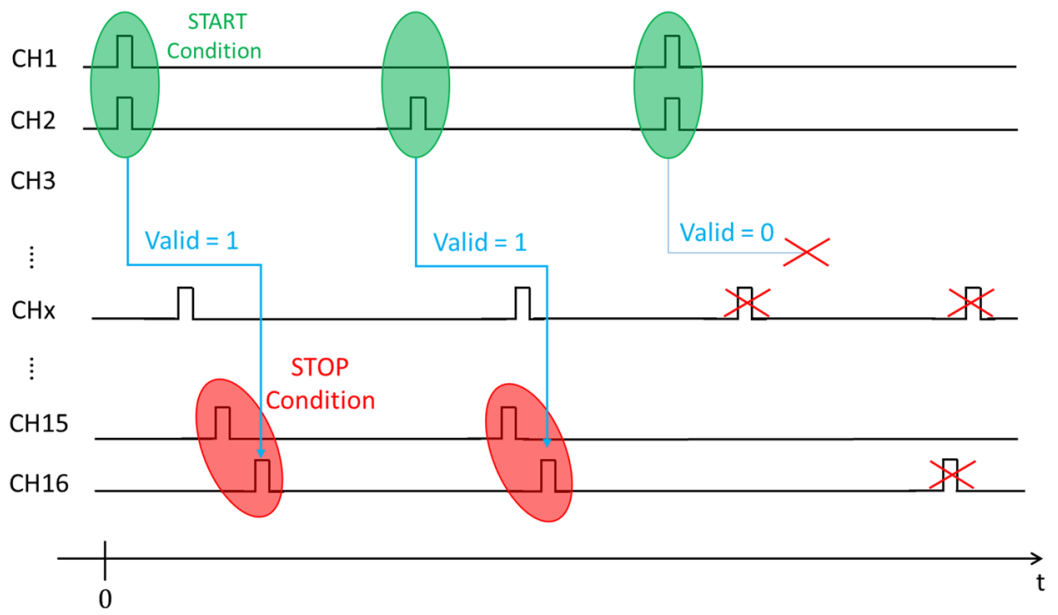

FIG. 6. Timing diagram of the acquisition with level-zero trigger. Channels 1 and $2(\mathrm{CH} 1, \mathrm{CH} 2)$ are in OR-mask, whereas channels 15 and $16(\mathrm{CH} 15, \mathrm{CH} 16)$ are in ANDmask defining START and STOP conditions, respectively.

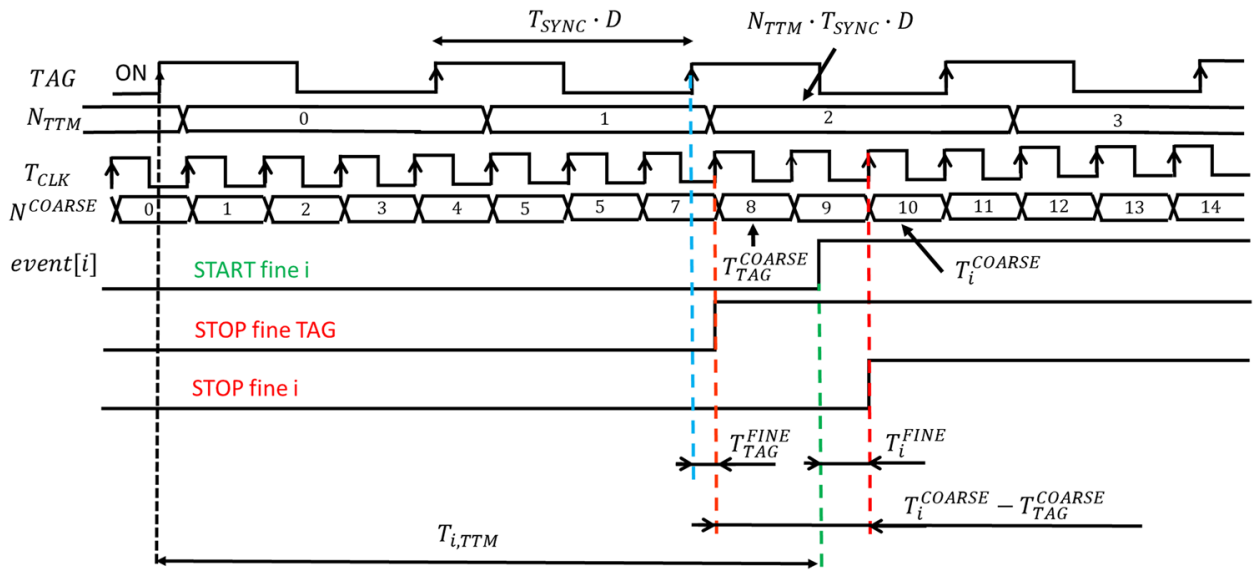

FIG. 7. Timing diagram of the calculation of the absolute time-tag $T_{i, \text { TTM }}$ of the ith event

the filtering effort processing only the class of timestamps required (Fig. 6).

Thanks to software flexibility, the Time-Tagging read-out Mode (TTM) ${ }^{48,27}$ extends the hardware full-scale range until several days at the maximum measuring rate of $1 \mathrm{MHz}$. In this module, the "acquisition efficiency" consists in getting the maximum dynamic range possible at the maximum resolution available. For doing this, an extra periodic signal called $S Y N C$, at frequency $f_{S Y N C}$, correlated with the input events, is introduced.

Generally, the SYNC is a very fast signal obtained, for instance, from the optical pulse train of a laser and is the absolute time reference of the events under measurement. Instead, the other input events are obtained by detection of the photons inside the optical pulses.

Since $f_{S Y N C}$ is fixed by an external source, the SYNC can be decimated of a tunable integer factor $D$ to get the TAG signal,

$$
f_{T A G}=f_{S Y N C} / D \text {. }
$$

The edges of the TAG signal increment a 48-bit counter $N_{T T M}$ synchronous with $T_{C L K}$. Since it is impossible to lock in phase with

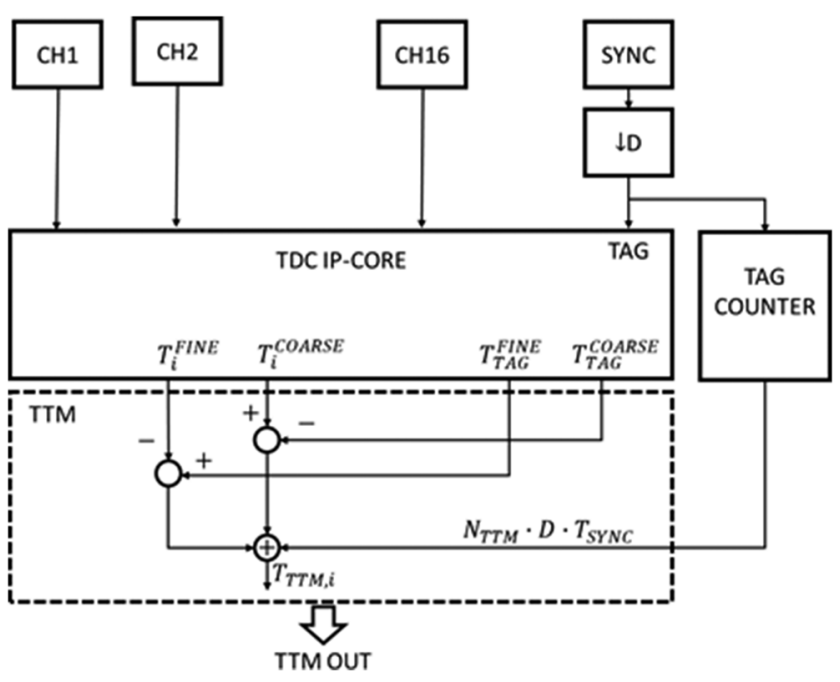

FIG. 8. Block diagram of TTM firmware. 


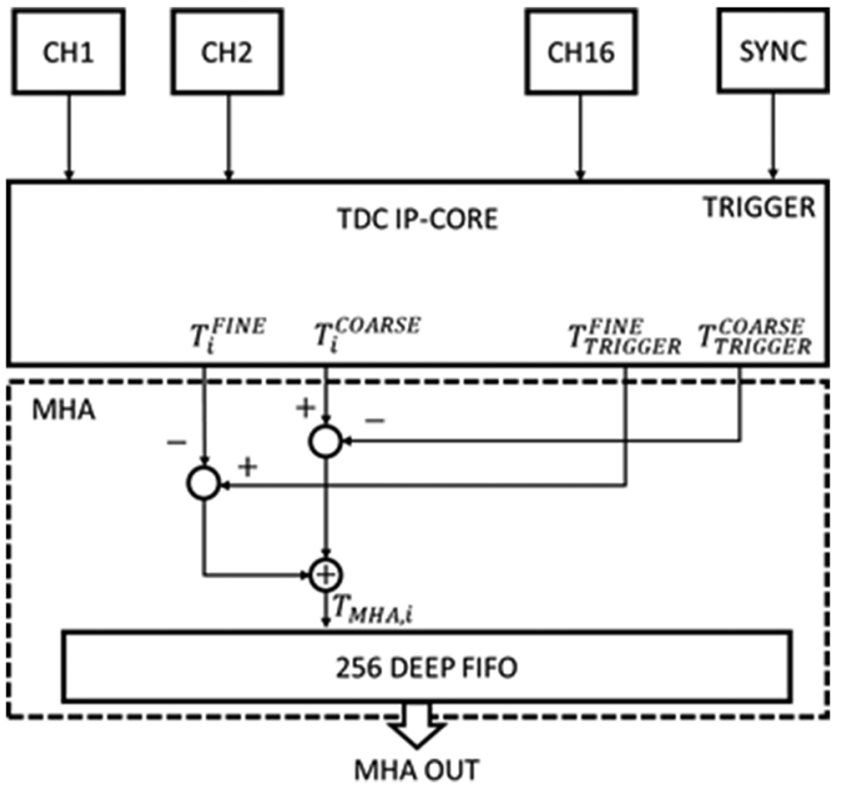

FIG. 9. Block diagram of MHA firmware.

the necessary precision the SYNC with the clock of TDC IP-core $\left(T_{C L K}\right)$, an ancillary channel of measure is requested. It measures the time position $\left(T_{T A G}{ }^{C O A R S E}\right.$ and $T_{\text {TAG }}{ }^{\text {FINE }}$ ) from the rising edge of the TAG (i.e., the decimated $S Y N C$ ) with respect to the rising edge of $T_{C L K}$ (Fig. 7). Consequently, the components of the timestamp of the $i$ th event $\left(T_{i}{ }^{C O A R S E}\right.$ and $\left.T_{i}{ }^{F I N E}\right)$ are corrected by the measurement of the reference TAG edge $\left(T_{T A G}{ }^{C O A R S E}\right.$ and $\left.T_{\text {TAG }}{ }^{F I N E}\right)$, and the absolute time-tag $\left(T_{T T M, i}\right)$ is

$T_{T T M, i}=N_{T T M} \cdot T_{S Y N C} \cdot D+\left(T_{i}^{C O A R S E}-T_{T A G}^{C O A R S E}\right)+\left(T_{T A G}^{F I N E}-T_{i}^{F I N E}\right)$.

Figure 8 shows the block diagram of the TTM. The timestamps produced by the TDC IP-core are managed as reported in
Eq. (5). For maximizing the performance, arithmetic operations are implemented in DSP block resources. The TTM is necessary when the not filtered acquisition of all the timestamps generated over an acquisition time bigger than the full-scale range is requested. Furthermore, the TTM is fundamental for the identification of very complex patterns that cannot be detected by the LZT. The huge amount of data sent and stored to the PC limits the transfer rate to $1 \mathrm{Mmeasures/s.}$

The third option is the MultiHits Acquisition (MHA), which has the same structure of TTM, except for the absence of $N_{T T M}$ (Fig. 9). The MHA acquires at the maximum rate of $20 \mathrm{MHz}$ per channel all the timestamps in a user-defined window smaller than the full-scale range of the TDC IP-core $(157 \mu \mathrm{s})$ after a trigger that substitutes the TAG (Fig. 10). Consequently, for performing the measurement, the components of the timestamp of the $i$ th event $\left(T_{i}^{C O A R S E}\right.$ and $\left.T_{i}^{F I N E}\right)$ are corrected by those of the trigger $\left(T_{\text {TRIGGER }}{ }^{\text {COARSE }}\right.$ and $\left.T_{\text {TRIGGER }}^{\text {FINE }}\right)$, i.e.,

$$
T_{M H A, i}=\left(T_{i}^{C O A R S E}-T_{T R I G G E R}^{C O A R E}\right)+\left(T_{\text {TRIGGER }}^{F I N E}-T_{i}^{F I N E}\right) .
$$

In order to sustain the maximum rate, measures are temporarily saved in a BRAM inside the FPGA capable to store 256 values of them and transferred to the host PC after the acquisition is completed.

The MHA is a lite and faster version of the TTM used when a higher acquisition rate and a lower full-scale range are required. In this context, "acquisition efficiency" is the maximization of measuring rate and resolution. In fact, the MHA allows us to record all the timestamps produced in FPGA without transmitting them to the PC. Moreover, the use of the MHA is necessary instead of the TTM if the SYNC signal that stimulated the generation of the events is not perfectly periodic or correlated with the input events. However, only the time distances inside the full-scale range of the TDC are measurable.

\section{Software}

The software is a Windows application developed in Visual Studio and performs the storage and processing of measures from

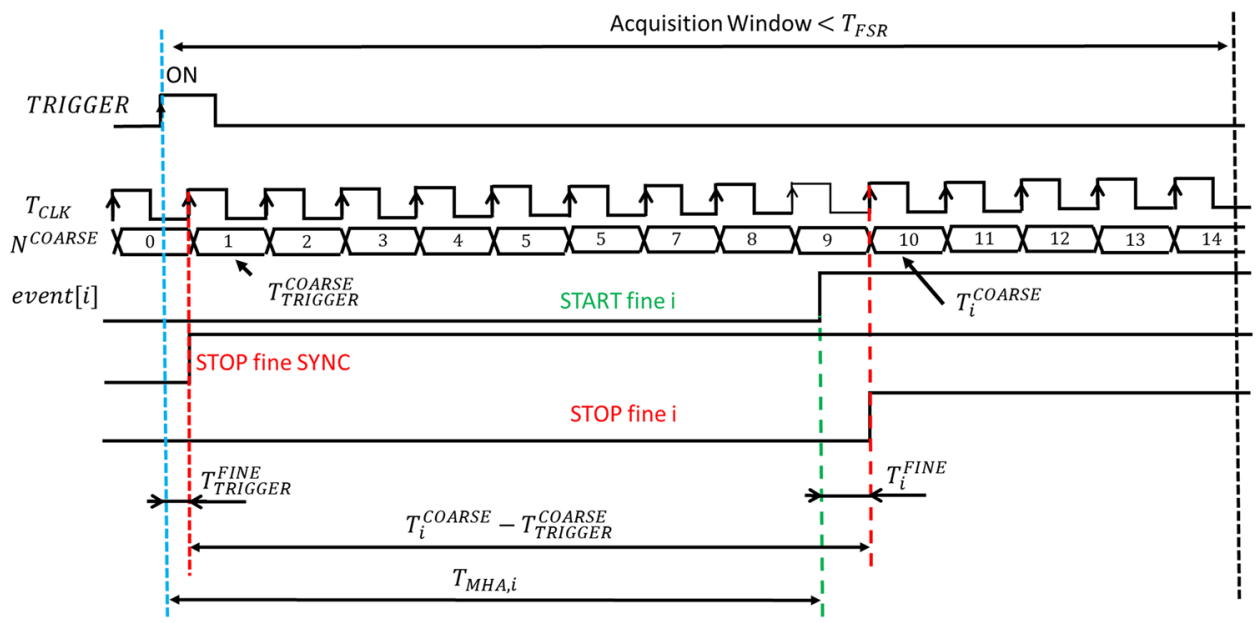

FIG. 10. Timing diagram of the MultiHit Acquisition $T_{M H A, i}$ of the ith event. 


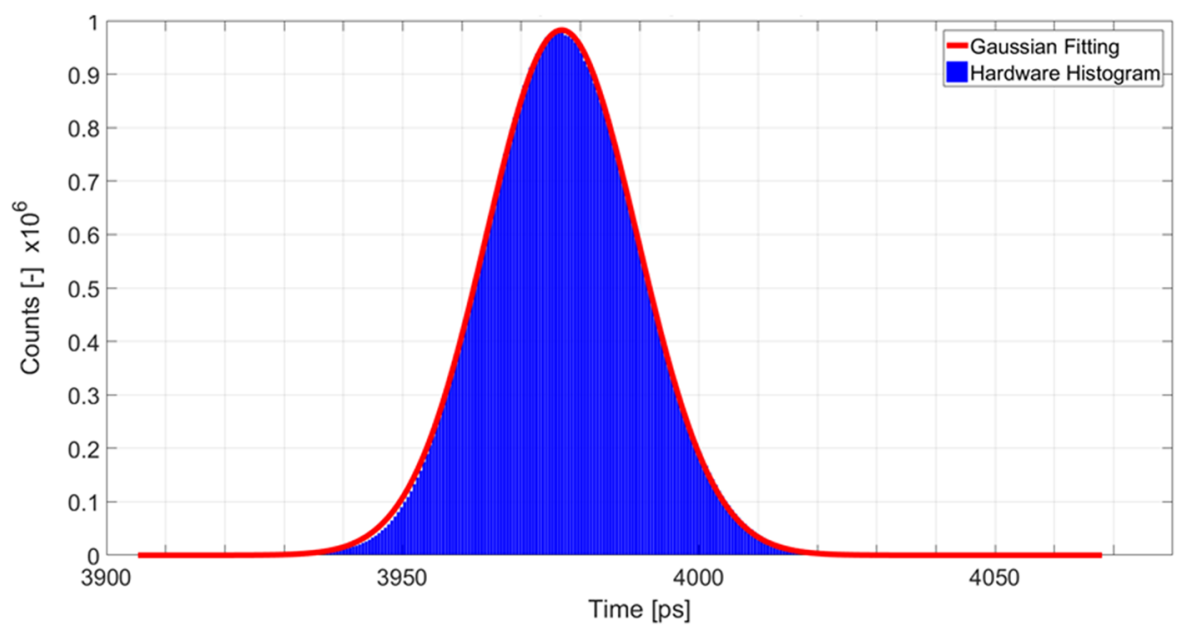

FIG. 11. Timing histogram calculated by the Hardware Histogram-Maker Module. The histogram is computed over $10^{\circ}$ measures with a bin width of 2 ps (resolution, LSB). The shape of the timing statistics is Gaussian with very high confidence $(\mathrm{R} 2<0.9996)$, featuring a standard deviation of $12.8 \mathrm{ps}$ rms that means a channel precision of $9.1 \mathrm{ps}$ rms (precision). the FPGA. Moreover, the same application allows programming the setting of the firmware and controlling the calibration procedure.

The software is structured in the same modular way as the firmware and allows monitoring in real-time data from level-zero trigger, time-tagging module, and multihit acquisition modules and storing the selected timestamps.

Of particular relevance is the hardware histogram-maker module that calculates the histogram of timestamps in FPGA (Fig. 11). The module accepts and manages measures in the pipeline mode up

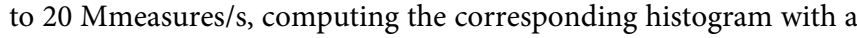
tunable bin width.

Thanks to an intuitive graphic interface, the application makes the instrument very user-friendly.

\section{SYSTEM CHARACTERIZATION}

The characterization of the instrument has been carried out not only in laboratory but also in the fieldwork of several research projects.

TABLE III. Features and performance of the considered version of the instrument.

\begin{tabular}{lc}
\hline \hline Feature & Value \\
\hline Number of channels & 16 \\
TDC clock period & $2.4 \mathrm{~ns}$ \\
System frequency & $100 \mathrm{MHz}$ \\
Interpolation technique & SuperWU \\
Device occupation per channel & $110 \mathrm{~ns}$ \\
Channel processing latency & $20 \mathrm{MHz} /$ channel \\
Maximum channel rate of measuring & $2 \mathrm{ps}$ \\
Total channel resolution (LSB) & $<12.5 \mathrm{ps} \mathrm{rms}$ \\
Total channel precision & $157 \mu \mathrm{s}$ \\
Full scale range & $<4.2 \mathrm{ps}$ \\
Maximum nonlinearity error & $5 \mathrm{~V}, 2 \mathrm{~A}$ \\
Power supply & Not detectable \\
Voltage drift influence & $286 \mathrm{fs} /{ }^{\circ} \mathrm{C}$ without the Peltier cell \\
Temperature drift influence & $<3 \mathrm{ps}$ rms only on active channels \\
Channels cross talk & $10 \mathrm{Mmeasures} / \mathrm{s}$ \\
Precision cross talk influence & 1 Mmeasures $/ \mathrm{s}$ \\
Max. timestamp transfer rate LZT & 15 days \\
Max. timestamp transfer rate TTM & 256 \\
Time-tagging full scale-range & {$[0 ; 3.3] \mathrm{V}$} \\
Max. measurements MHA & 3 ns \\
Input signal amplitude range & \\
Minimum input pulse width & No evidence both on idle and active channels \\
\hline
\end{tabular}



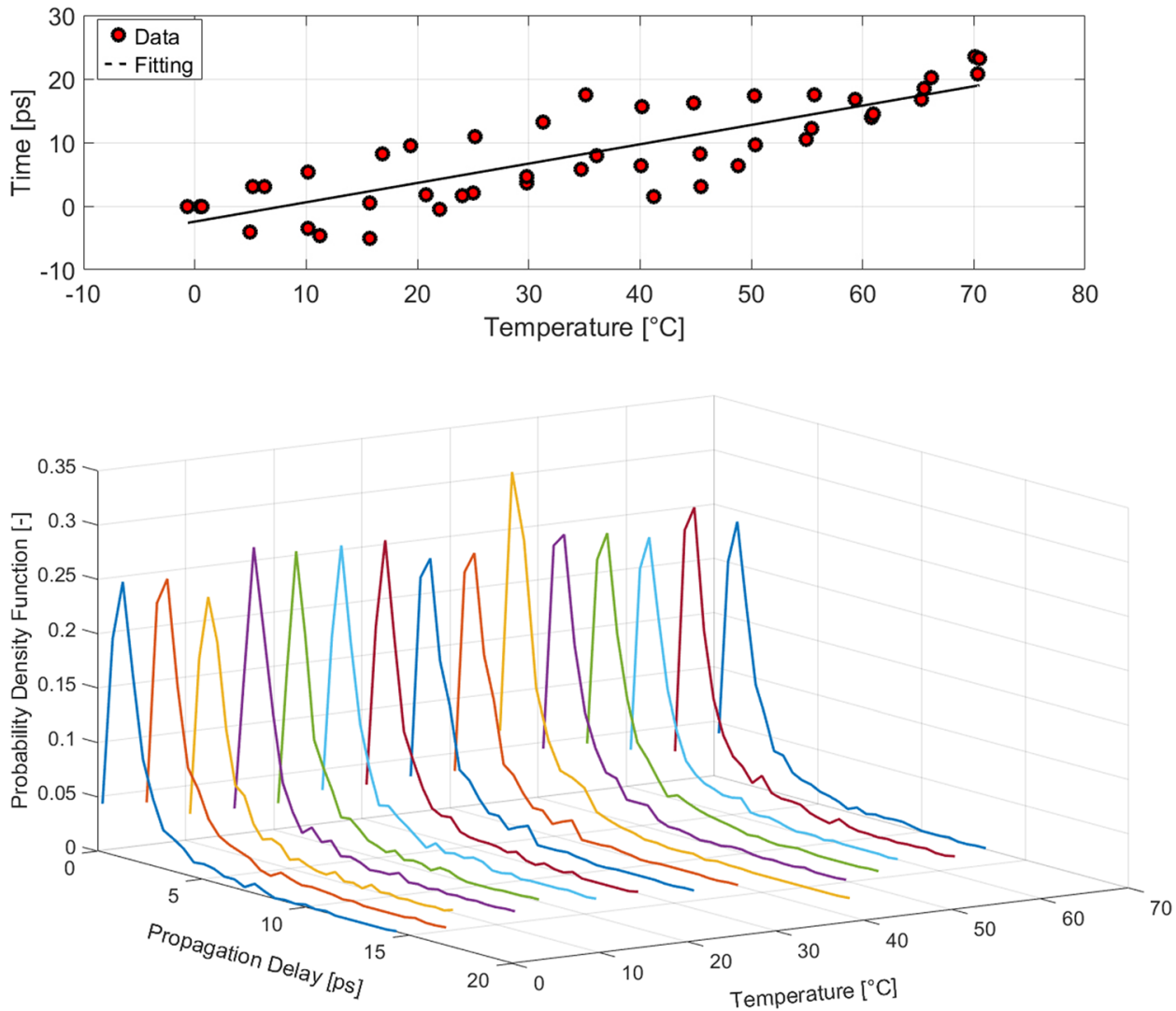

FIG. 12. Dependence on the temperature of mean values of measures of the same interval.
As mentioned above, the instrument considered for this presentation is equipped with a Xilinx Artix $^{\circledR}-7$ XC7A200T-1 FPGA (33 650 SLICEs, 13 140-kbit BRAM, 740 DSPs) in the package of area $19 \times 19 \mathrm{~mm}^{2} .49,47$

\section{A. Performance}

Table III gives a synoptic view of the main features of the instrument from the functional point of view.

\section{B. Temperature effects}

We have specifically investigated the dependence on temperature without any thermostatic action of precision and mean value of the measures, in an extended operative range between $0{ }^{\circ} \mathrm{C}$ and $70{ }^{\circ} \mathrm{C}$.

Over the whole range, the drift of the measurement values of the same intervals was $20 \mathrm{ps}\left(286 \mathrm{fs} /{ }^{\circ} \mathrm{C}\right)$, which is comparable with the system precision (Fig. 12). This means that in a reasonable operative range of $\pm 10^{\circ} \mathrm{C}$ around room temperature, the thermal drift of measures is reduced to about \pm 2.86 ps that is negligible with respect to the system precision ( $<12.5 \mathrm{ps} \mathrm{rms})$.

Experiments have put in evidence that also other characteristic parameters of the implemented subinterpolated SuperWU V-TDL are not significantly affected by temperature variations. Figure 13 shows the probability density function that describes the dispersion of the propagation delay of the 1200 virtual bins that comprise the subinterpolated SuperWU V-TDL as functions of the temperature in a range from $0{ }^{\circ} \mathrm{C}$ to $70{ }^{\circ} \mathrm{C}$.

\section{Linearity}

We have experimentally verified that the worst case of integral nonlinearity of the instrument is always below 4.2 ps over $35 \mathrm{~ns}$ of full-scale range.

Figure 14 shows the integral nonlinearity error as a function of the time interval measured. The curve was obtained by the

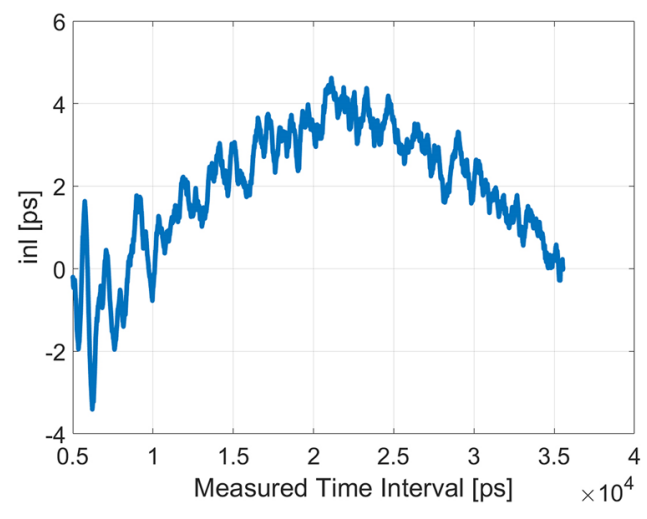

FIG. 14. Measurement of integral nonlinearity. 
TABLE IV. Comparison of the most relevant figures of merit of the proposed instrument with respect to two significant ASIC solutions.

\begin{tabular}{lccc}
\hline \hline Feature & Proposed instrument & Reference 53 & Reference 54 \\
\hline TDC channel resolution (LSB) (ps) & 2 & 5.5 & 10 \\
TDC channel precision (psrms) & $<12.5$ & $<4.95$ & $<21.3$ \\
Full-scale range (s) & $157 \mu \mathrm{s}$ & $90.1 \mathrm{~ns}$ & $160 \mu \mathrm{s}$ \\
Maximum channel rate (MHz) & 20 & $\mathrm{~N} . \mathrm{A}$. & 6.7 \\
Number of channels & 16 & 1 & 3 \\
Technological node (nm) & 28 & 40 & 350 \\
Reconfigurability & Yes & No & \\
\hline \hline
\end{tabular}

TABLE V. Comparison of the most relevant figures of merit of the proposed instrument with respect to reference commercial solutions.

\begin{tabular}{lccc}
\hline \hline Feature & Proposed & $\begin{array}{c}\text { HydraHarp 400 } \\
\text { (PicoQuant) }\end{array}$ & $\begin{array}{c}\text { TimeHarp 260 } \\
\text { PICO (PicoQuant) }\end{array}$ \\
\hline Discriminator & Threshold prog. $(50 \mu \mathrm{V})$ & CFD & CFD \\
Input signal amplitude (V) & {$[0 ; 3.3]$} & {$[0 ; 1]$} & {$[0 ; 1.2]$} \\
TDC channel resolution (LSB) (ps) & 2 & 1 & 2 \\
TDC channel precision (ps rms) & $<12.5$ & $<12$ & $<20$ \\
Full-scale range (s) & $157 \mu \mathrm{s}$ & 2.9 & 17.1 \\
Maximum channel rate (MHz) & 20 & 12.5 & 40 \\
Number of channels & 16 & 8 & 25 \\
Channel processing latency (ns) & 110 & 4 & 2 \\
Total power consumption (W) & $<10$ & $<100$ & $<15$ \\
Reconfigurability & Yes & No & No \\
\hline \hline
\end{tabular}

integration and equalization of a density code histogram composed of $1.5 \times 10^{10}$ counts normalized in picoseconds. The $1.5 \times 10^{10}$ start and stop events were obtained, respectively, by a $3.33 \mathrm{MHz}$ square wave and a completely uncorrelated single photon avalanche diode (SPAD) operating at the rate of $100 \mathrm{kHz}$. The low rate of the SPAD with respect to the frequency of the stop events and the big value of the counts guarantee a uniform investigation of the code density.

\section{Comparison with ASIC solutions}

The performance of the proposed instrument and of customized solutions implemented in specific integrated digital circuits is pretty much the same. The main advantage in our case is that the instrument is fully programmable.

Table IV compares the proposed instrument to a couple of particularly significant ASIC solutions of TDCs for different target applications, i.e., PLL $^{50}$ and measurement.

Table V compares the proposed instrument to very well-known instruments at the state-of-the-art, whose TDC processing cores are implemented in ASICs.

\section{APPLICATIONS}

The applications for debugging, testing, and engineering the instrument in many research projects have also highlighted its extreme flexibility and reconfigurability in the most different experimental conditions.

Just to name a few of these applications, high-resolution/ precision and multichannel features were fundamental in the characterization of the timing jitter of array of detectors such as Super Nanowire Simple Photon Detectors (SNSPDs), ${ }^{52}$ Single Photon Avalanche Diode (SPAD), and Silicon Photo Multipliers (SiPMs). Moreover, the high full-scale range made the instrument suitable for several kinds of experiments based on time-of-fly measures in the LHCb collaboration ${ }^{54}$ and for electron beam tracking measures. ${ }^{55}$ At last, an achievable high channel rate was essential in photon statistic experiments. $^{30}$

Fundamental in several of these applications was the availability of different acquisition modes, thus fulfilling requirements of different experimental conditions and detectors used.

\section{CONCLUSIONS}

A fully configurable high-performance time-resolving instrument based on subinterpolated SuperWU has been realized and tested.

The system can be configured with up to 16 channels operating in parallel with a resolution of 2 ps (intended as LSB of the timestamps), precision below 12.5 ps r.m.s, over a hardware full-scale-range of $157 \mu$ s. 
The possibility of using different acquisition modes (LZT, TTM, and MHA) allows us to manage in different ways the timestamps provided by the TDC IP-core without reducing resolution and precision.

In particular, the level-zero trigger module makes possible to filter all the timestamps in real-time, sustaining a total transfer rate

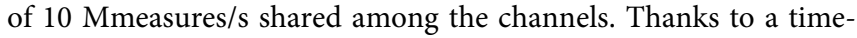
tagging module, the extension of the full-scale range to some days is feasible simply operating via software. Moreover, a MultiHit Acquisition module allows storing 256 measures at the maximum rate of $20 \mathrm{MHz}$ per each channel.

The firmware and the software of the instrument are modularly structured, meaning that each module has firmware and software images that split the processing in parallel and temporal segments.

By changing the plugged-in processing module, the instrument can be reconfigured also in its hardware structure.

The instrument has been characterized in temperature without any thermostatic action in an operative range from $0{ }^{\circ} \mathrm{C}$ to $70{ }^{\circ} \mathrm{C}$, showing only a drift of $286 \mathrm{fs} /{ }^{\circ} \mathrm{C}$ of the mean value of the measures. At last, the instrument guarantees linearity error below 4.2 ps over $35 \mathrm{~ns}$ of dynamic range.

\section{ACKNOWLEDGMENTS}

The authors are thankful to Edoardo Charbon of EPFL and TU Delft, Gabriele Bulgarini of Single Quantum BV, Christophe Galland of EPFL, and Giuseppe Cautero of Elettra Sincrotrone Trieste for the fieldwork activity in debugging, testing, and engineering the instrument.

\section{REFERENCES}

${ }^{1}$ V. Ravinuthula, V. Garg, J. G. Harris, and J. A. B. Fortes, "Time-mode circuits for analog computation," Int. J. Circuit Theory Appl. 37(5), 631-659 (2009).

${ }^{2}$ S. Donati, Electro-Optical Instrumentation (Prentice Hall, Upper Saddle River, 2010).

${ }^{3}$ Edinburgh Instruments Ltd, What is TCSPC, Edinburgh Photonics, 2012.

${ }^{4}$ D. V. O'Connor and D. Phillips, Time-Correlated Single Photon Counting (Academic Press, London, 1984).

${ }^{5}$ R. J. Cotter, "Time-of-flight mass spectrometry for the structural analysis of biological molecules," Anal. Chem. 64, 1027A (1992).

${ }^{6}$ J. R. Lakowicz, Principles of Fluorescence Spectroscopy (Springer US, 2006).

${ }^{7} \mathrm{~K}$. Maatta and J. Kostamovaara, "A high-precision time-to-digital converter for pulsed time-of-flight laser radar applications," IEEE Trans. Instrum. Meas. 47, 521-536 (1998).

${ }^{8}$ T. Beyer, D. W. Townsend, J. Czernin, and L. S. Freudenberg, "The future of hybrid imaging-Part 2: PET/CT,” Insights Imaging 2, 225-234 (2011).

${ }^{9} \mathrm{~V}$. Unnikrishnan and M. Vesterbacka, "Time-mode analog-to-digital conversion using standard cells," IEEE Trans. Circuits Syst. I 61, 3348-3357 (2014).

${ }^{10}$ Z. M. H. Saleh, R. Al-Araji, and M. A. Al-Qutayri, Digital Phase Lock Loops (Springer US, 2006).

${ }^{11}$ S. Cova and M. Bertolaccini, "The logic design of high precision time-to-pulseheight converters. Part I. General problems and logic structure of a converter," Nucl. Instrum. Methods 121, 547-556 (1974).

${ }^{12}$ D. Resnati, I. Rech, A. Gallivanoni, and M. Ghioni, "Monolithic time-toamplitude converter for time correlated single photon counting," Rev. Sci. Instrum. 80(8), 086102 (2009).

${ }^{13}$ F. Yuan, CMOS Time-Mode Circuit Systems (CRC Press, 2015).

${ }^{14}$ G. G. Shahidi, "Challenges of CMOS scaling at below $100 \mathrm{~nm}$," in ICM 2000 Proceedings of 12th International Conference on Microelectronics, Tehran, Iran, 31 October-2 November 2000 (IEEE, 2000), pp. 5-8.
${ }^{15}$ R. J. Gera and D. H. K. Hoe, "An evaluation of CMOS adders in deep submicron processes," in Proceedings of 44th Southeastern Symposium on System Theory (SSST), March 2012.

${ }^{16}$ B. E. Jonsson, "On CMOS scaling and A/D-converter performance," NORCHIP, Tampere, Finland, 2010 (IEEE, 2010), pp. 1-4.

${ }^{17}$ F. Yuan, CMOS Current-Mode Circuits for Data Communications (Springer US, 2007).

${ }^{18}$ M. Beretta, P. Ciambrone, M. Gatta, F. Gonnella, L. Iafolla, M. Mascolo, R. Messi, D. Moricciani, D. Riondino, and A. Balla, "The characterization and application of a low resource FPGA-based time to digital converter," Nucl. Instrum. Methods Phys. Res., Sect. A 739, 75-82 (2014).

${ }^{19} \mathrm{~J}$. Wu and Z. Shi, "The 10-ps wave union TDC: Improving FPGA TDC resolution beyond its cell delay," in IEEE Nuclear Science Symposium Conference Record (IEEE, 2008), pp. 3440-3446.

${ }^{20} \mathrm{C}$. Favi and E. Charbon, "A 17ps time-to-digital converter implemented in 65nm FPGA technology," in Proceedings of the 7th ACM SIGDA International Symposium on Field-Programmable Gate Arrays, Monterey, California, USA, 2009, pp. 113-120.

${ }^{21}$ F. Caponio, A. Abba, N. Lusardi, and A. Geraci, "A high-precision Wave Union TDC implementation in FPGA," in Proceedings of IEEE Nuclear Science Symposium (IEEE, COEX, Seoul, Korea, 2013).

${ }^{22} \mathrm{M}$. Traxler and E. Bayer, "A high-resolution (<10 ps RMS) 32-channel timeto-digital converter (TDC) implemented in a field programmable gate array (FPGA)," IEEE Trans. Nucl. Sci. 58(4), 1-5 (2011).

${ }^{23} \mathrm{M}$. Traxler and E. Bayer, "A high-resolution (<10 ps RMS) 48-channel timeto-digital converter (TDC) implemented in a field programmable gate array (FPGA)," IEEE Trans. Nucl. Sci. 58(4), 1547 (2011).

${ }^{24}$ G. Korcyl, J. Michel, M. Penschuk, M. Traxler, and C. Ugur, "264 channel TDC platform applying 65 channel high precision (7.2 ps RMS) FPGA based TDCs," in Proceedings of IEEE Nordic-Mediterranean Workshop on Time-to-Digital Converters (NoMe TDC) (IEEE, Perugia, Italy, 2013).

${ }^{25}$ S. Bourdeauducq, "Time to digital converter core for spartan 6 FPGAs," Open Hardware Repository, November 2011.

${ }^{26}$ M. W. Fishburn, L. H. Menninga, C. Favi, and E. Chardon, "A 19.6 ps, FPGAbased TDC with multiple channels for open source applications," IEEE Trans. Nucl. Sci. 60, 2203-2208 (2013).

${ }^{27} \mathrm{M}$. Wahl, Time-Correlated Single Photon Counting (PicoQuant GmbH, Berlin, Germany, 2016).

${ }^{28}$ L. Stebel, M. Malvestuto, V. Capogrosso, P. Sigalotti, B. Ressel, F. Bondino, E. Magnano, G. Cautero, and F. Parmigiani, "Time-resolved soft x-ray absorption setup using multi-bunch operation modes at synchrotrons," Rev. Sci. Instrum. 82(12), 123109 (2011).

${ }^{29}$ E. Venialgo, N. Lusardi, F. Garzetti, A. Gerac, S. Brunner, E. Schaart, R. Dennis, and E. Charbon, "Towards a full-flexible and fast-prototyping TOF-PET block detector based on TDC-on-FPGA," in IEEE Transactions on Radiation and Plasma Medical Sciences (IEEE, 2018).

${ }^{30}$ N. Lusardi, J. W. N. Los, R. B. M. Gourgues, G. Bulgarini, and A. Geraci, "Photon counting with photon number resolution through superconducting nanowires coupled to a multi-channel TDC in FPGA," Rev. Sci. Instrum. 88(3), 035003 (2017).

${ }^{31}$ H. Gong, Y. Liu, and J. Qi, "On-chip real-time correction for a 20-ps wave union time-to-digital converter (TDC) in a field-programmable gate array (FPGA)," IEEE Trans. Nucl. Sci. 59(4), 1605 (2012).

${ }^{32}$ N. Lusardi and A. Geraci, "Eight-channels high-resolution TDC in FPGA," in Proceedings of IEEE Nuclear Science Symposium (IEEE, San Diego, USA, 2015).

${ }^{33} \mathrm{C}$. Lui and Y. Wang, "A 128-channel, $710 \mathrm{M}$ samples/second, and less than $10 \mathrm{ps}$ RMS resolution time-to-digital converter implemented in a kintex-7 FPGA," IEEE Trans. Nucl. Sci. 62(3), 773-783 (2015).

${ }^{34}$ S. Berrima, Y. Blaquière, and Y. Savaria, "A multi-measurements RO-TDC implemented in a Xilinx field programmable gate array," in IEEE International Symposium on Circuits and Systems (ISCAS) (IEEE, Baltimore, MD, 2017), pp. 1-4.

${ }^{35}$ M. A. Daigneault and J. P. David, "Towards 5 ps resolution TDC on a dynamically reconfigurable FPGA," in Proceedings of 18th Annual ACM/SIGDA 
International Symposium on Field Programmable Gate Arrays, Monterey, California, USA, 2010.

${ }^{36}$ X. Qin, C. Feng, D. Zhang, M. Deliang, B. Miao, L. Zhao, X. Hao, S. Liu, and Q. An, "Development of a high resolution TDC for implementation in flash-based and anti-fuse FPGAs for aerospace application," IEEE Trans. Nucl. Sci. 60(5), 3550-3556 (2013).

${ }^{37}$ J. Wang, S. Liu, Q. Shen, H. Li, and Q. An, "A fully fledged TDC implemented in field-programmable gate arrays," IEEE Trans. Nucl. Sci. 57(2), 446-450 (2010). ${ }^{38} \mathrm{~L}$. Zhao, X. Hu, S. Liu, J. Wang, and Q. An, "The design of a 16-channel 15 ps TDC implemented in a $65 \mathrm{~nm}$ FPGA," IEEE Trans. Nucl. Sci. 60(5), 3532-3536 (2013).

${ }^{39}$ M. Daigneault and J. P. David, "A novel 10 ps resolution TDC architecture implemented in a $130 \mathrm{~nm}$ process FPGA," in Proceedings of 8th IEEE International NEWCAS Conference (IEEE, Montreal, QC, 2010).

${ }^{40}$ N. Lusardi, A. Abba, F. Caponio, and A. Geraci, "Quantization noise in nonhomogeneous calibration table of a TCD implemented in FPGA," in Proceedings of 2014 IEEE Nuclear Science Symposium (IEEE, Seattle, USA, 2014).

${ }^{41}$ N. Lusardi, F. Garzetti, and A. Geraci, "The role of sub-interpolation for delayline time-to-digital converters in FPGA devices," Nucl. Instrum. Methods Phys. Res., Sect. A 916, 204-214 (2019).

${ }^{42}$ J. David and M. Daigneault, "A high-resolution time-to-digital converter on FPGA using dynamic reconfiguration,” IEEE Trans. Instrum. Meas. 60(6), 2070 (2011).

${ }^{43} \mathrm{~N}$. Lusardi and A. Geraci, "Comparison of interpolation techniques for TDCs implementation in FPGA," in Proceedings of IEEE Nuclear Science Symposium (IEEE, San Diego, USA, 2015).

${ }^{44}$ L. Zhao, S. Liu, J. Wang, Q. An, and X. Hu, "A stepped-up tree encoder for the 10-ps wave union TDC," in Proceedings of IEEE-NPSS Real Time Conference (IEEE, Berkeley, CA, USA, 2012).

${ }^{45}$ Analog Devices, Inc., Rail-to-Rail, Very Fast, $2.5 \mathrm{~V}$ to $5.5 \mathrm{~V}$, Single-Supply LVDS Comparators, Norwood, MA, USA, 2006.

${ }^{46}$ Analog Devices, Inc., Octal, 12-/16-Bit nanoDAC + with $2 \mathrm{ppm} /{ }^{\circ} \mathrm{C}$ Reference, I2C Interface, Norwood, MA, USA, 2006.
${ }^{47}$ See https://www.trenz-electronic.de for Trenz Electronic GmbH.

${ }^{48}$ PicoQuant GmbH, Photon counting and timing, Berlin, Germany, 2016.

${ }^{49}$ See https://www.xilinx.com for Xilinx, Inc.

${ }^{50} \mathrm{~J}$. Borremans, K. Vengattaramane, V. Giannini, and J. Craninckx, "A $86 \mathrm{MHz}-$ to- $12 \mathrm{GHz}$ digital-intensive phase-modulated fractional-N PLL using a $15 \mathrm{pJ} / \mathrm{Shot}$ 5 ps TDC in $40 \mathrm{~nm}$ digital CMOS," in IEEE International Solid-State Circuits Conference-(ISSCC) (IEEE, San Francisco, CA, 2010).

${ }^{51}$ D. Tamborini, M. Buttafava, A. Ruggeri, and F. Zappa, "Compact, low-power and fully reconfigurable 10 ps resolution, 160 us range, time-resolved singlephoton counting system," IEEE Sens. J. 16(10), 3827-3833 (2016).

${ }^{52}$ N. Lusardi, F. Garzetti, G. Bulgarini, R. B. M. Gourgues, J. W. N. Los, and A. Geraci, "Single photon counting through multi-channel TDC in programmable logic," in Proceedings of IEEE Nuclear Science Symposium (IEEE, Strasbourg, France, 2016).

${ }^{53}$ E. Venialgo, N. Lusardi, A. Geraci, K. O’Neill, S. Gnecchi, C. Jackson, S. B. Brunner, D. R. Schaart, and E. Charbon, "An order-statistics inspired multichannel readout for analog SiPMs," in Proceedings of IEEE Nuclear Science Symposium (IEEE, Strasbourg, France, 2016).

${ }^{54}$ LHCb, "Observation of the Bs $0 \rightarrow \mathrm{J} / \psi \phi \phi$ decay," J. High Energy Phys. 2016, 40 (2016).

${ }^{55}$ N. Lusardi, F. Garzetti, A. Geraci, G. Cautero, C. Dri, P. Pittana, R. Sergio, and L. Stebel, "Development of fully FPGA-based 3D (X, Y,t) detection systems using multi-channel tapped delay-line time-to-digital converter with cross delay-line detectors," in Proceedings of Nuclear Science Symposium, Atlanta, USA, 2017.

${ }^{56}$ Q. Shen, S. Liu, B. Qi, Q. An, S. Liao, P. Shang, C. Peng, and W. Liu, "A 1.7 ps equivalent bin size and 4.2 ps RMS FPGA TDC based on multichain measurements averaging method," IEEE Trans. Nucl. Sci. 62(3), 947-954 (2015). ${ }^{57} \mathrm{Y}$. Wang and C. Liu, "A 4.2 ps time-interval RMS resolution time-to-digital converter using a bin decimation method in an UltraScale FPGA," IEEE Trans. Nucl. Sci. 63(5), 2632-2638 (2016).

${ }^{58}$ P. Chen, Y. Y. Hsiao, Y. S. Chung, W. X. Tsai, and J. M. Lin, “A 2.5-ps bin size and 6.7-ps resolution FPGA time-to-digital converter based on delay wrapping and averaging," IEEE Trans. VLSI Syst. 25(1), 114-124 (2017). 